\title{
Corrigendum: Coherent creation and destruction of orbital wavepackets in Si:P with electrical and optical read-out
}

K.L. Litvinenko, E.T. Bowyer, P.T. Greenland, N. Stavrias, Juerong Li, R. Gwilliam, B.J. Villis, G. Matmon, M.L.Y. Pang, B. Redlich, A.F.G. van der Meer, C.R. Pidgeon, G. Aeppli \& B.N. Murdin

Nature Communications 6:6549 doi: 10.1038/ncomms7549 (2015); Published 20 Mar 2015; Updated 20 Apr 2017

This Article was originally published under a CC BY-NC-ND 4.0 license, but has now been made available under a CC BY 4.0 license. The PDF and HTML versions of the paper have been modified accordingly. 